PACS 78.20.Ci, 78.20.-e, 78.40.-q

\title{
Complex index of refraction of indium nitride InN
}

\author{
J.O. Akinlami*, F.M. Bolaji \\ Department of Physics, University of Agriculture, P.M.B 2240, Abeokuta, Ogun State, Nigeria \\ *E-mail: johnsonak2000@yahoo.co.uk
}

\begin{abstract}
We have investigated the complex index of refraction of Indium Nitride (InN). We obtained refractive index which has the maximum value 2.59 at the photon energy $5.30 \mathrm{eV}$, the extinction coefficient which has the maximum value 0.86 at the photon energy $5.30 \mathrm{eV}$, the dielectric constant, the real part of the complex dielectric constant with the peak value 5.90 at the photon energy $5.30 \mathrm{eV}$ and the imaginary part of the complex dielectric constant with the maximum value 4.48 at the photon energy $5.30 \mathrm{eV}$, the transmittance with the maximum value 0.1402 at the photon energy $5.30 \mathrm{eV}$, the absorption coefficient which has its maximum value 86.0 at the photon energy $11.50 \mathrm{eV}$ and reflection coefficient which with the maximum value 0.49 at the photon energy $5.3 \mathrm{eV}$. Thus, InN has the potential to operate optimally in a photonic device at the photon energy $5.30 \mathrm{eV}$.
\end{abstract}

Keywords: complex index of refraction, extinction coefficient, complex dielectric constant, transmittance, absorption coefficient, reflection coefficient, photon energy.

Manuscript received 08.02.12; revised version received 10.05.12; accepted for publication 14.06.12; published online 25.09.12.

\section{Introduction}

When one of the group III elements, boron, aluminum, gallium or indium is bonded to the group $\mathrm{V}$ element nitrogen, a III-nitride compound semiconductor is formed. Different combinations of group III-V elements have produced more than twenty-five (25) III-V compound semiconductors, examples of which are indium nitride $(\mathrm{InN})$, gallium arsenide $(\mathrm{GaAs})$, gallium nitride $(\mathrm{GaN})$, aluminum nitride (AIN). Group III nitride are now a widely studied class of semiconductor materials and they have found commercial success in the last fifteen years as light emitting diodes and lasers in the green to near-ultraviolet regions of the electromagnetic spectrum [1-8]. In comparison to $\mathrm{GaN}$ and AlN, InN remains one of the least understood Nitride semiconductors. The lack of attention to $\mathrm{InN}$ was mainly due to the following two reasons: first, high quality single crystalline InN is difficult to grow and secondly, there exist alternative, well characterized semiconductors such as GaAs and AlGaAs which have energy band gaps close to $1.89 \mathrm{eV}$ [9], the band gap previously associated with InN. For more than thirty years, the band gap of indium nitride has been stated to be in the 1.8 to $2.0 \mathrm{eV}$ range. However, recent measurements [10-11] using more reliable growth processes and higher crystal quality material have reported a value of $0.7 \mathrm{eV}$. The discovery of this lower band gap value, together with the smallest effective mass, the largest mobility and highest peak and saturation velocities of the III-nitrides, has the potential to lead to a variety of novel device applications, including high-efficiency multi-junction solar cells, high-brightness multi-colour light emitting diodes and high electron mobility transistors [12-14].

The higher refractive index of InN compared to $\mathrm{GaN}$ and $\mathrm{AlN}$ is one of the important aspects of using it for photonic band egde design [15]. The use of InNbased optoelectronic devices offers the potential of an environmentally friendly red emitter with no toxic elements to replace GaAs-based devices. In addition, InN is a potential material for transparent conducting windows used in heterojunction tandem solar cells [16]. InN thin films reveal a potential application as chemical and biological sensors. The electrical response of $\mathrm{InN}$ surface to chemical exposure demonstrated that InN surface shows a fast response to certain solvent exposure with a large increase in surface carrier density. Other 
potential applications of $\mathrm{InN}$ are in fabrication of metaloxide semiconductor field effect transistor (MOSFET) devices [17], anodes for lithium-ion thin film batteries [18] and thermoelectric devices [19, 20].

In this work, we have investigated complex index of refraction of indiun nitride $(\mathrm{InN})$.

\section{Method of calculation}

The reflection coefficient measures the fractional amplitude of the reflected electromagnetic field, and it is the square root of reflectance:

$r=\sqrt{R}$,

where $r$ is the reflection coefficient and $R$ - reflectance. The reflectance spectrum of $\mathrm{InN}$ measured at the angle of incidence $\theta(\omega)=15^{\circ}$ obtained by Sobolev and Zlobina [21] was used to obtain the reflection coefficient using Eq. (1). We carried out the Kramers-Kronig analysis of the reflectivity data to obtain the refractive index and the extinction coefficient using Eqs (2) and (3).

$$
\begin{aligned}
& n(\omega)=\frac{1-r^{2}(\omega)}{1+r^{2}(\omega)-2 r(\omega) \cos \theta(\omega)}, \\
& k(\omega)=\frac{2 r(\omega) \sin \theta(\omega)}{1+r^{2}(\omega)-2 r(\omega) \cos \theta(\omega)} .
\end{aligned}
$$

The complex dielectric constant is a fundamental intrinsic property of material. The real part of the dielectric constant shows how much it will slow down the speed of light in the material, whereas the imaginary part shows how a dielectric material absorbs energy from an electric field due to dipole motion. The knowledge of the real and the imaginary parts of dielectric constant provides information about the loss factor which is the ratio of the imaginary part to the real part of the dielectric constant. The real and imaginary parts of the dielectric constant can be estimated using the relations [22]:

$$
\begin{aligned}
& E_{1}=n^{2}-k^{2}, \\
& E_{2}=2 n k .
\end{aligned}
$$

The absorption coefficient $(\alpha)$ can be calculated using the equation

$$
\alpha=\frac{4 \pi k}{\lambda}
$$

where $k$ is the extinction coefficient, and $\lambda$ - wavelength.

The transmittance is obtained from the relation

$R+T+A=1$,

where $R, T$ and $A$ represent the reflectance, transmittance and absorbance respectively. The sum of these macroscopic quantities usually known as the optical properties of the material must equal unity since the incident radiant flux at one wavelength is distributed totally between reflected, transmitted and absorbed intensity. The absorbance $A$ is given by

$$
A=\log \left(\frac{1}{R}\right) \text {. }
$$

\section{Results and discussion}

The refractive index obtained for $\mathrm{InN}$ within the photon energy range 2.0 to $20.0 \mathrm{eV}$ is shown in Fig. 1. Variation of the refractive index $n$ with photon energy shows six peaks at photon energies $2.30,5.30,7.90,8.90,10.0$, and $11.20 \mathrm{eV}$. The refractive index for InN varied from 1.35 to 2.59. It was observed that the refractive index has its maximum value 2.59 at the photon energy $5.30 \mathrm{eV}$, and it is greater than unity over the entire energy range. As can be seen from Fig. 1, the refractive index first increases with photon energy and then decreases with increase in it. This decrease in refractive index indicates that InN shows normal dispersion behaviour. Values of the refractive index are not constant over the entire photon energy range, and thus it implies a photon energy dependence. The refractive index decreases as the photon energy increases. Therefore, the refractive index is a function of wavelength, and this effect is known as chromatic dispersion, and this is what causes a prism to divide white light into its constituent spectra colours, explain rainbows and also is the cause of chromatic aberration in lenses. Thus InN can also be used as a material in making the lenses. The speed of the photon in this material within the photon energy range 2.0 $13.0 \mathrm{eV}$ is lesser compared to that in the energy range 13.0 to $20.0 \mathrm{eV}$, due to higher absorption in the energy range 2.0 to $13.0 \mathrm{eV}$ compared to that of the energy range 13.0 to $20.0 \mathrm{eV}$. The value of the refractive index obtained is in good agreement with that reported by Tyagai et al. (2.56) [23] and Silva Pinto et al. (2.6) [24].

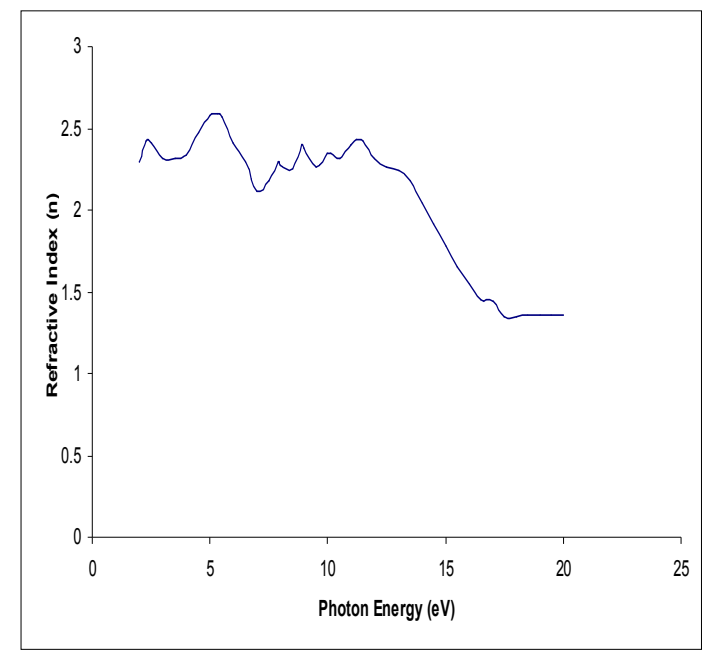

Fig. 1. Refractive index of indium nitride. 


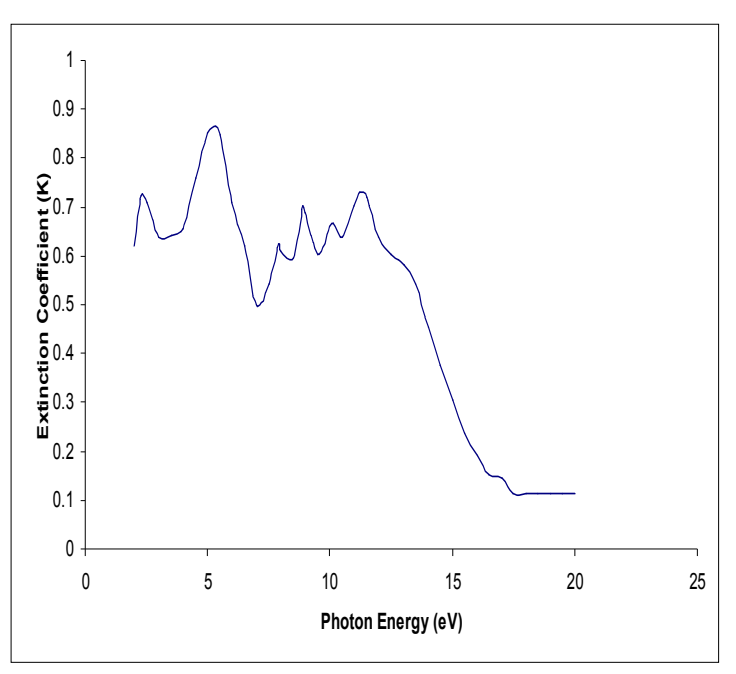

Fig. 2. Extinction coefficient of indium nitride.

The extinction coefficient obtained for $\mathrm{InN}$ in the photon energy range $2.0-20.0 \mathrm{eV}$ is shown in Fig. 2 . Variation of the extinction coefficient with photon energy shows six distinct peaks at photon energies 2.30 , 5.30, 7.90, 8.90, 10.10, and $11.20 \mathrm{eV}$. The extinction coefficient varied from 0.11 to 0.86 . It was observed that the extinction coefficient has its maximum value 0.86 at the photon energy $5.30 \mathrm{eV}$ and it decreases gradually until becoming the constant. The extinction coefficient is non-zero, which means that $\mathrm{InN}$ is not transparent in this energy region. It was observed that at low photon energy the extinction coefficient is high and at higher photon energy it is low and decays to a constant. It shows that the extinction coefficient is photon energy dependent. It can be seen from Fig. 2, that the extinction coefficient decreases with increase in the photon energy. The decrease in extinction coefficient with increase in photon energy shows that the fraction of light lost due to scattering and absorbance decreases. Also, the peaks indicate regions of deep penetration of the electromagnetic wave. This penetration of the electromagnetic wave decreases as the extinction coefficient values approaches the peaks and consequently, the absorption loss also increases with these peaks.

The real part of the complex dielectric constant $\varepsilon_{1}$ obtained for Indium Nitride ( $\mathrm{InN}$ ) in the photon energy range $2.0-20.0 \mathrm{eV}$ is shown in Fig. 3. Variation of the real part of complex dielectric constant, $\varepsilon_{1}$, with photon energy shows six distinct peaks at photon energies 2.30, $5.30,7.90,8.90,10.10$, and $11.20 \mathrm{eV}$. It was observed that the real part of complex dielectric constant $\varepsilon_{1}$ has a maximum value $\varepsilon_{1}=5.90$ at the photon energy $5.30 \mathrm{eV}$, and it decreases gradually until becoming a constant. It was also observed that the real part of the complex dielectric constant decreases with increase in photon energy. This shows that the loss factor decreases with increase in photon energy. The real part of the complex dielectric constant is positive over the entire energy region, that is, $\bar{n}>\bar{K}$. The physical significance or meaning of this is that electromagnetic wave in this region incident on $\mathrm{InN}$ can propagate through it. The maxima correspond to higher propagation of electromagnetic waves. At higher photon energy, the propagation of the electromagnetic wave drops drastically, thus InN tends to become an insulator at this energy range.

The imaginary part of the complex dielectric constant, $\varepsilon_{2}$, obtained for $\mathrm{InN}$ in the energy range 2.0 to $20.0 \mathrm{eV}$ is shown in Fig. 4. Variation of the imaginary part $\varepsilon_{2}$ for the dielectric constant with photon energy shows six distinct peaks at 2.30, 5.30, 7.90, 9.0, 10.10, and $11.20 \mathrm{eV}$. It was observed that the imaginary part of the complex dielectric constant $\varepsilon_{2}$ for $\mathrm{InN}$ has its maximum value $\varepsilon_{2}=4.48$ at the photon energy $5.30 \mathrm{eV}$, and it decreases gradually until it becomes a constant. The propagation of electromagnetic waves is faster at the peak energy values, this reduces for higher photon energies (from 13.0 up to $18.0 \mathrm{eV}$ ) and then becomes constant from 18.0 up to $20.0 \mathrm{eV}$. It shows that the

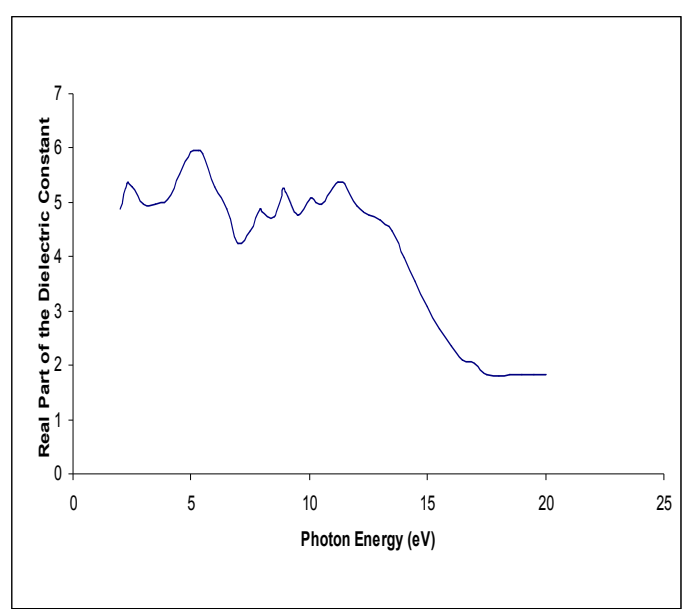

Fig. 3. Complex dielectric (real part) of indium nitride.

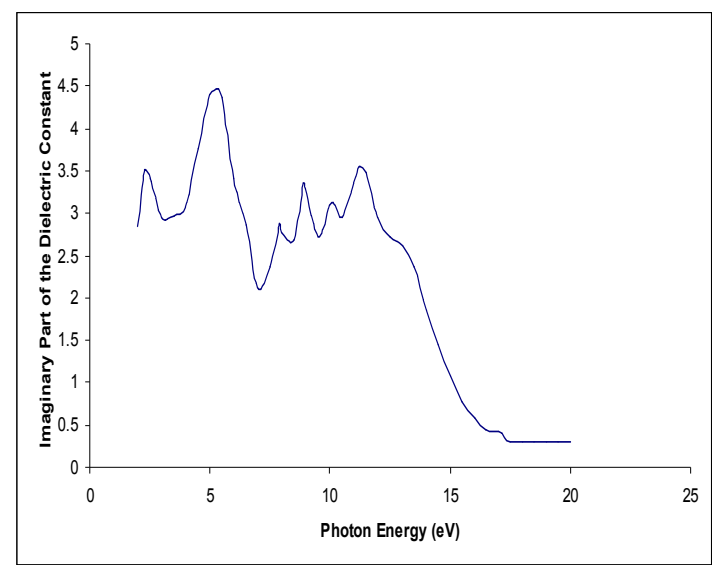

Fig. 4. Complex dielectric constant (imaginary oart) of indium nitride. 
imaginary part of the complex dielectric constant is high at low photon energies and low at high photon energies, and this shows that the imaginary part of the complex dielectric constant has a photon energy dependence. As the photon energy increases, the imaginary part of the complex dielectric decreases. This shows that the loss factor decreases with increase in the photon energy. The value of the imaginary part of dielectric constant obtained is close to that reported by Silva Pinto et al. (5.0) [24].

The transmittance $(T)$ obtained for $\mathrm{InN}$ within the energy range 2.0 to $20.0 \mathrm{eV}$ is shown in Fig. 5. Variation of transmittance with the photon energy shows six peaks at photon energies $2.30,5.30,7.90,8.90,10.10$, and $11.20 \mathrm{eV}$. It was observed that the transmittance has its maximum value 0.1402 at the photon energy $5.30 \mathrm{eV}$, and it decreases gradually to zero. For high photon energies there is no transmission because all the light is absorbed. For low photon energies, transmission is high in this region. With the maximum 0.1402 for transmittance, it means that $\mathrm{InN}$ is not a good transmitter of electromagnetic waves in this energy region. At the photon energy $14.0 \mathrm{eV}$, the transmittance value is equal to zero, which means that no electromagnetic wave is transmitted in this region.

The absorption coefficient $\alpha$ obtained for $\mathrm{InN}$ in the photon energy range 2.0 to $20.0 \mathrm{eV}$ is shown in Fig. 6 . Variation of the absorption coefficient with photon energy shows six peaks at photon energies 5.50, 7.90, $9.00,10.10,11.50$, and $13.60 \mathrm{eV}$. It was observed that the absorption coefficient $\alpha$ has its maximum value 86 at the photon energy $11.50 \mathrm{eV}$. This high value of the absorption coefficient is typical for interband absorption in semiconductors [25]. It was observed that the absorption coefficient increases with increase in the photon energy within the range 2.0 to $11.5 \mathrm{eV}$ and then decreases afterwards. The energy at which the absorption starts was just a little above its direct band gap at $1.92 \mathrm{eV}$. InN shows no absorption below its band gap. The degree of absorption depends among other things on the number of free electron capable of receiving the photon energy. At the maximum absorption coefficient, the electromagnetic wave is observed more. Thus, indium nitride absorbs more at these energy values.

The reflection coefficient for InN obtained in the photon energy range $2-20 \mathrm{eV}$ is shown in Fig. 7. Variation of the reflection coefficient with photon energy also shows six peaks at the photon energies 2.3, $5.3,7.9,8.9,10.0$, and $11.20 \mathrm{eV}$. It was observed that the reflection coefficient has its highest value 0.49 at the photon energy $5.30 \mathrm{eV}$, and a sharp decrease happens subsequently. Absorption becomes more intense, and the reflection coefficient decreases accordingly. Our value for the reflection coefficient agreed with that of Silva Pinto et al. (0.49) [24]. The result obtained shows that at low photon energy the reflection coefficient is high, and at higher photon energies the reflection coefficient is low and decays to a constant.

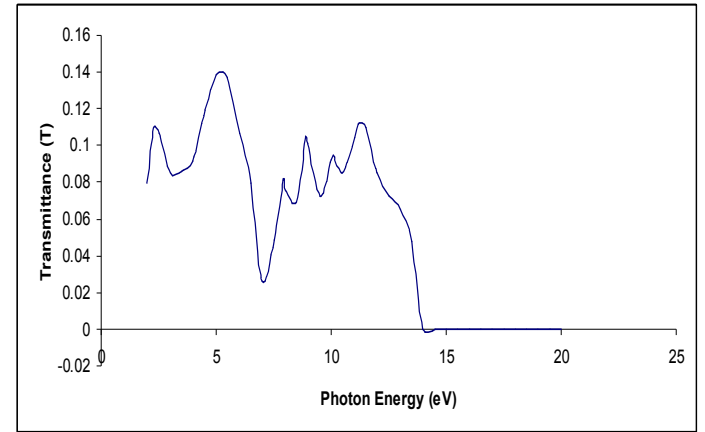

Fig. 5. Transmittance of indium nitride.

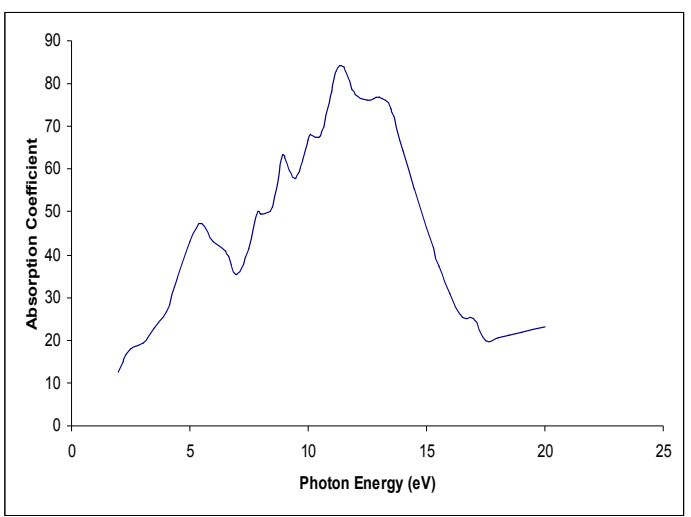

Fig. 6. Absorption coefficient of indium nitride.

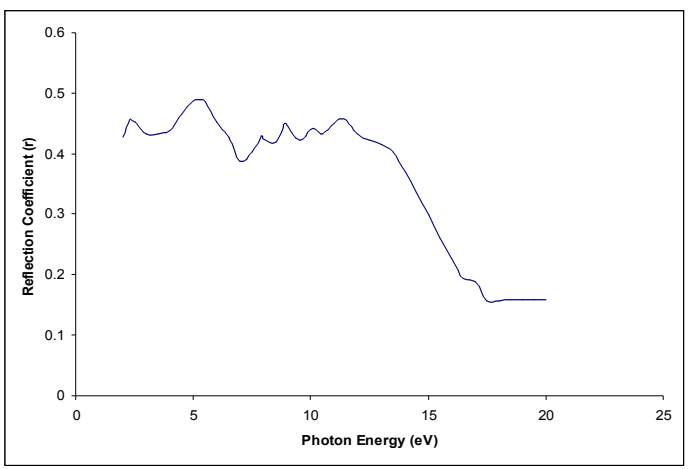

Fig. 7. Reflection coefficient of indium nitride.

\section{Conclusion}

We have investigated theoretically the complex index of refraction of Indiun Nitride ( $\mathrm{InN}$ ) within the energy range $2.0-20.0 \mathrm{eV}$. The refractive index $n$ obtained for $\mathrm{InN}$ in this energy range shows six peaks at energies $2.30,5.30,7.90,8.90,10.00$, and $11.20 \mathrm{eV}$. The refractive index obtained has its maximum value $n=2.59$ at the photon energy $5.30 \mathrm{eV}$. The value of the refractive index obtained is in agreement with that reported by Tyagai et al. and Silva Pinto et al. The extinction coefficient obtained for InN shows six distinct peaks at the photon energies 2.30, 5.30, 7.90, 8.90, 10.10 , and $11.20 \mathrm{eV}$. The extinction coefficient obtained has its maximum value $k=0.86$ at the photon energy $5.30 \mathrm{eV}$. The refractive index and the extinction 
coefficient decreased with increase in the photon energy. The decrease in refractive index with increase in the photon energy indicates that $\mathrm{InN}$ shows normal dispersion behaviour. The decrease in extinction coefficient with increase in photon energy shows that the fraction of light lost due to scattering and absorbance decreases. The real part of the complex dielectric constant obtained for $\mathrm{InN}$ in the photon energy range 2.0-20.0 eV shows six distinct peaks at photon energies $2.30,5.30,7.90,8.90,10.10$, and $11.20 \mathrm{eV}$. The real part of the complex dielectric constant obtained has its maximum value 5.90 at the photon energy $5.30 \mathrm{eV}$, and it decreases gradually until it becomes a constant. The real part of the complex dielectric constant is positive over the entire energy region. The maxima are regions of higher propagation of the electromagnetic wave. At higher photon energies, propagation of electromagnetic waves drops drastically, thus InN tends to become an insulator in this energy range. The imaginary part of the complex dielectric constant obtained for $\mathrm{InN}$ in the energy range $2.0-20.0 \mathrm{eV}$ shows six distinct peaks at $2.30,5.30,7.90,9.0,10.10$, and $11.20 \mathrm{eV}$. The imaginary part of the complex dielectric constant for InN has its maximum value 4.48 at the photon energy $5.30 \mathrm{eV}$, and it is in agreement with that reported by Silva Pinto et al. The real and imaginary parts of the dielectric constant decrease with increase in photon energy. These results indicate that for InN the loss factor decreases with increase in the photon energy. The transmittance obtained for InN within the photon energy range 2.0 to $20.0 \mathrm{eV}$ shows six peaks at the photon energies 2.30, 5.30, 7.90, 8.90, 10.10, and $11.20 \mathrm{eV}$. The transmittance has its maximum value 0.1402 at the photon energy $5.30 \mathrm{eV}$. With the maximum value 0.1402 for transmittance, it means that $\mathrm{InN}$ is not a good transmitter of electromagnetic waves in this energy region. The absorption coefficient obtained for $\mathrm{InN}$ in the energy range $2.0-20.0 \mathrm{eV}$ shows six peaks at the photon energies 5.30, 7.90, 9.0, 10.10, 11.50, and $13.60 \mathrm{eV}$. The absorption coefficient has its maximum value 86.0 at the photon energy $11.50 \mathrm{eV}$ which is typical for interband absorption in semiconductors. The reflection coefficient for $\mathrm{InN}$ obtained in the photon energy range 2 to $20 \mathrm{eV}$ shows six peaks at the photon energies $2.3,5.3,7.9,8.9,10.0$, and $11.20 \mathrm{eV}$. It was observed that the reflection coefficient has its maximum value 0.49 at the photon energy $5.30 \mathrm{eV}$, and a sharp decrease happens subsequently. Absorption becomes more intense, and the reflection coefficient decreases accordingly. Our value for the reflection coefficient agrees with that of Silva Pinto et al.

\section{References}

1. S. Nakamura, M. Senoh, S. Nagahma, N. Iwasa, T. Yamada, T. Matsuahita, H. Kiyoku, and Y. Sugimoto // Jpn. J. Appl. Phys. 35, p. 174 (1996).
2. T. Lei, T.D. Moustakas, R.J. Graham, Y. He, and S.J. Berkowitz // J. Appl. Phys. 71, p. 4933 (1992).

3. S.J. Hwang, W. Shan, R.J. Hauenstein, and J.J. Song // Appl. Phys. Lett. 64, p. 2928 (1994).

4. A. Rubio, J.L. Corkill, M.L. Cohen, E.L. Shirley and S.G. Louie // Phys. Rev. B, 48, 11810 (1993).

5. E.A. Albanesi, W.R.L. Lambrecht, and B. Segall // Phys. Rev. B, 48, 17841(1993).

6. A.F. Wright and J.S. Nelson // Phys. Rev. B, 50, p. 2159 (1994).

7. S. Strite and H. Morkoc // J. Vac. Sci. Technol. B, 10, p. 1237 (1992).

8. P.R. Bhatta, Electron Spectroscopic Study of Indium Nitride Layers. Ph.D. Thesis, College of Arts and Science, Georgia State University (2008).

9. T.L. Tansley and C.P. Foley // J. Appl. Phys. Rev. 59, p. 3241(1986).

10. V.Y. Davydov, A.A. Klochikhin, R.P. Seisyan et al. // Phys. Status Solidi B, 229 (2002).

11. J. Wu, W. Walukiewicz, K.M. Yu, et al. // Appl. Phys. Lett. 80, p. 3967 (2002)

12. B.E. Frutz, S.K. O'Leary, M.S. Shur and L.F. Eastman // J. Appl. Phys. 85, p. 7727 (1999).

13. J. Wu, W. Walukiewicz, K.M. Yu et al. // J. Appl. Phys. 94, p. 6477 (2003).

14. S. Keller, I. Benyaacov, S.P. Denvers and UK. Mishra. Proc. Intern. Workshop on Nitride Semiconductors IPAP Conf., series 1, 233 (Nagoya, Japan, 2000).

15. M. Leroux, B. Il, J.H. Edger, S. Strite, I. Akasaki, H. Amano, C. Wetzel, Gallium Nitride and Related Semiconductors. Publ. INSPEC, 1999.

16. A. Yamanato, M. Tsujino, M. Ohkubo, A. Hashimoto // Sol. Energy Mater. Sol. Cells, 35 (1994).

17. H. Lu, W.J. Schaff, L.F. Eastman // Mater. Res. Soc. Symp. Proc. 693 (2002).

18. B.J. Neudecker, R.A. Zuhr // Electrochem. Soc. Proc. 99, p. 295 (2000).

19. S. Yamaguchi, R. Izaki, N. Kaiwa, S. Sugimura, A. Yamamoto // Appl. Phys. Lett. 84, p. 5344 (2004).

20. S. Yamaguchi, R. Izaki, K. Yamagiwa, K. Taki, Y. Iwamura, A. Yamamoto Appl. Phys. Lett. 83, p. 5398 (2003).

21. V.V. Sobolev and M.A. Zlobina // Electronic and Optical Properties of Semiconductors, 33, No.4, p. 385 (1999).

22. A. Goswami, Thin Film Fundamentals. New Age International, New Delhi, 2005.

23. V.A. Tyagai, V.A. Eustignev, A.M. Krasiko, A.F. Andreeva, V.Y. Malatidio // Semiconductors, 11, p. 1257 (1977).

24. E. Silva Pinto, R. de Paiva, L.C. de Carvalho, H.W.L. Alves and J.L.A. Alves // Microelectronics J. 34, p. 721 (2003).

25. M.D. Sturge // Phys. Rev. 127, p. 768 (1962). 